\title{
Anesthesiological management of laparoscopic surgery during the second trimester of pregnancy. Case Report
}

\author{
Heredia C, Gine M, Parrilla L, Estadella J, Moral MV. \\ Hospital de la Santa Creu i Sant Pau \\ Dept of Anaesthesiology, Barcelona Spain
}

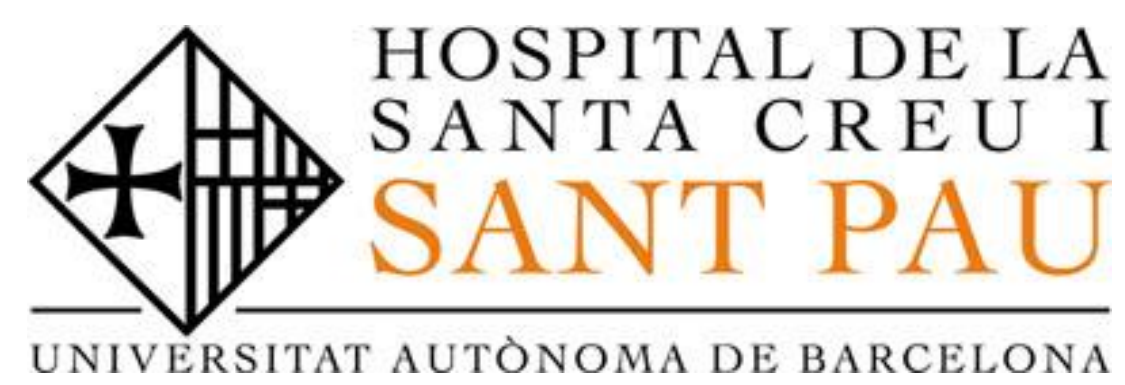

\section{BACKGROUND}

The frequency with which pregnancies are complicated by the need for non-obstetric surgical procedures is of the order of $0.75 \%$ to $2.0 \%[1,2]$. The range and incidence of non-obstetric surgical conditions encountered in pregnant patients are similar to those in the general population [2].

Although the advantages that laparoscopy approach offers during the pregnancy are well documented, it becomes an anesthesiological challenge concerning anatomic and physiologic changes related to pregnancy and anesthetic and surgical effects on the fetus.

\section{CASE REPORT}

A 34 year-old woman on her 19th week of gestation, without relevant medical history, was admitted at the emergency room of our hospital due to acute onset abdominal pain.

With this provisional diagnosis an ultrasound scan (US) was requested. The US revealed the presence of free abdominal fluid in the abdominal c vcavity probably due to hemoperitoneum (figure 1). Initial blood tests showed low hemoglobin levels and high leucocytes levels, with normal platelet count and coagulation parameters. In successive blood counts a progressive drop of the hemoglobin was recorded. In order to identify the cause and control the bleeding an explorative laparoscopy was performed.

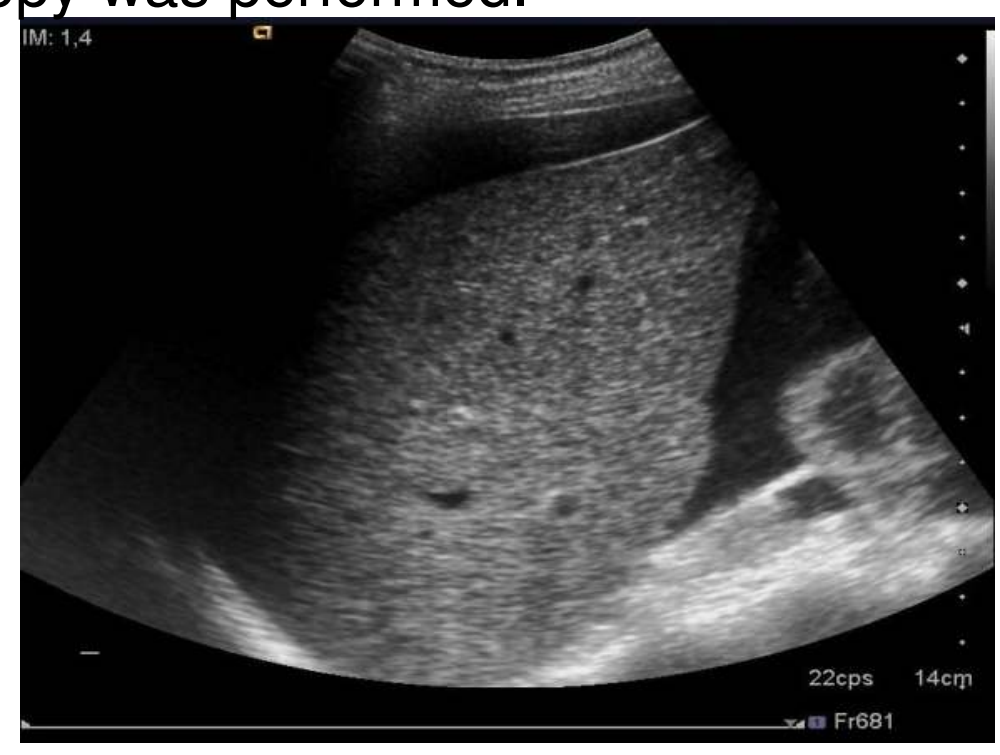

Figure 1. Free abdominal fluid. US scan image.
Rapid sequence intubation was conducted with propofol, rocuronium and fentanil. Total intravenous anesthesia was performed as maintenance and hemodynamic stability was constant. Low pressure maintained during the pneumoperitoneum at $8-12 \mathrm{mmHg}$. Maternal endtidal $\mathrm{CO}_{2}$ was within $29-35 \mathrm{mmHg}$ range. Inspection of the abdominal cavity found fresh venous blood due to a crumbly vascular network located on the anterior uterus wall (figure 2), where hemostasis was correctly performed. The patient was extubated without incidences and was admitted to the reanimation care unit.

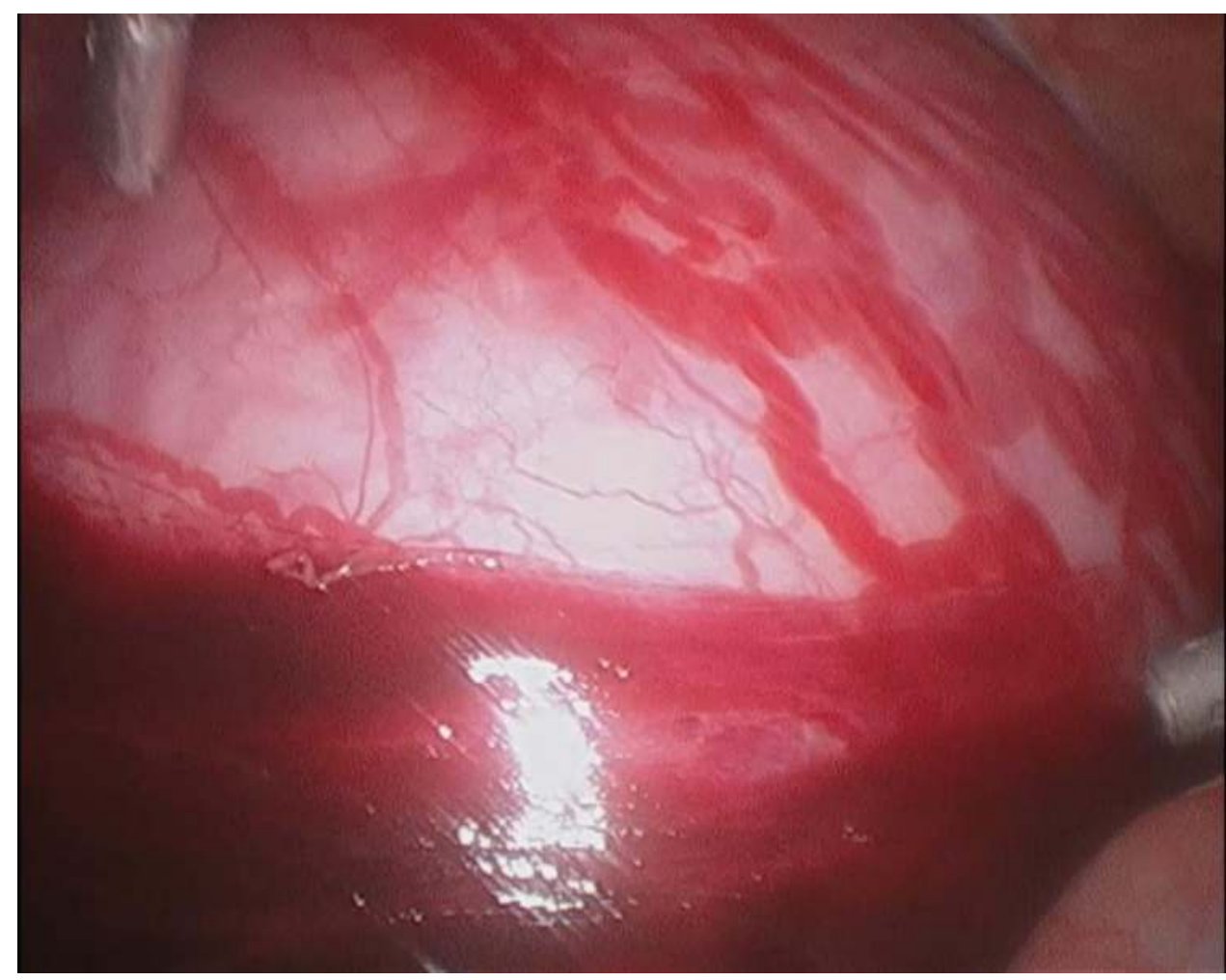

Figure 2. Vascular network at anterior uterus wall.

\section{CONCLUSION}

This case exemplifies that the laparoscopic approach is the technique of choice in non-obstetric surgery during the second trimester.

- Factors important in maternal laparoscopy are: monitor maternal end-tidal $\mathrm{CO}_{2}$ to avoid fetal acidosis, mantain low pneumoperitoneum pressure, limit the extent of Trendelenburg position and initiate any position slowly.

- The chief goal in the management of anesthesia is maintenance of uteroplacental perfusion. 